\title{
Short-Term VA Health Care Expenditures Following a Health Risk Assessment and Coaching Trial
}

\author{
Caroline Sloan, $M D^{1,2}$, Karen M. Stechuchak, MS , Maren K. Olsen, PhD ${ }^{1,3}$, \\ Eugene Z. Oddone, $\mathrm{MD}^{1,2}$, Laura J. Damschroder, $M S, M P H^{4,5}$, and \\ Matthew L. Maciejewski, PhD ${ }^{1,2,6}$ (])
}

'Durham Center of Innovation to Accelerate Discovery and Practice Transformation (ADAPT), , Durham Veterans Affairs Health Care System, Durham, NC, USA; ${ }^{2}$ Division of General Internal Medicine, Department of Medicine, Duke University, Durham, NC, USA; ${ }^{3}$ Department of Biostatistics and Bioinformatics, Duke University, Durham, NC, USA; ${ }^{4}$ Ann Arbor VA HSR\&D/Center for Clinical Management Research, Ann Arbor, MI, USA; ${ }^{5}$ A Diabetes QUERI, Ann Arbor, MI, USA; ${ }^{6}$ Department of Population Health Sciences, Duke University, Durham, NC, USA.

BACKGROUND: Short-term health care costs following completion of health risk assessments and coaching programs in the VA have not been assessed.

OBJECTIVE: To compare VA health care expenditures among veterans who participated in a behavioral intervention trial that randomized patients to complete a HRA followed by health coaching (HRA + coaching) or to complete the HRA without coaching (HRA-alone).

DESIGN: Four-hundred seventeen veterans at three Veterans Affairs (VA) Medical Centers or Clinics were randomized to HRA + coaching or HRA-alone. Veterans randomized to HRA-alone $(n=209)$ were encouraged to discuss HRA results with their primary care team, while veterans randomized to HRA + coaching $(n=208)$ received two brief telephone-delivered health coaching calls.

PARTICIPANTS: We included 411 veterans with available cost data.

MAIN MEASURES: Total VA health expenditures 6 months following trial enrollment were estimated using a generalized linear model with a gamma distribution and log link function. In exploratory analysis, model-based recursive partitioning was used to determine whether the intervention effect on short-term costs differed among any patient subgroups.

KEY RESULTS: Most participants were male (85\%); mean age was 56 , and mean body mass index was 34 . From the generalized linear model, 6-month estimated mean total VA expenditures were similar (\$8665 for HRA + coaching vs $\$ 9900$ for HRA-alone, $p=0.25)$. In exploratory subgroup analysis, among unemployed veterans with good sleep and fair or poor perceived health, mean observed expenditures in the HRA + coaching group were higher than in the HRA-alone group (\$12,814 vs \$7971). Among unemployed veterans with good sleep and good general health, mean observed expenditures in the HRA + coaching group were lower than in the HRA-alone group (\$5082 vs \$11,612).

CONCLUSIONS: Compared to completing and receiving HRA results, working with health coaches to set actionable health behavior change goals following HRA completion did not reduce short-term health expenditures.

Prior Presentations None.

Received June 20, 2019

Accepted September 27, 2019

Published online January 2, 2020
TRIAL REGISTRATION: Clinicaltrials.gov identifier: NCT01828567

KEY WORDS: veterans; behavioral; health care costs; expenditures.

J Gen Intern Med 35(5):1452-7

DOI: $10.1007 / \mathrm{s} 11606-019-05455-Z$

(C) Society of General Internal Medicine (This is a U.S. government work and not under copyright protection in the U.S.; foreign copyright protection may apply) 2019

\section{INTRODUCTION}

Health risk assessments (HRA) are increasingly being offered to employees of large corporations to help predict future health risks. ${ }^{1}$ HRAs are often coupled with health management programs (HMPs) that provide coaching or wellness programs to help employees act to reduce their health risks. The goal of HRAs and HMPs is to improve the health of the employee pool while simultaneously reducing the health care costs for which employers are responsible. ${ }^{2}$ There is evidence to suggest that employees become more acutely aware of their modifiable risks and tend to change their behavior accordingly when they complete HRAs. ${ }^{3}$ As a result, their utilization of appropriate care (i.e., outpatient visits) increases, ${ }^{4,5}$ and utilization of expensive care (i.e., hospitalizations) decreases. ${ }^{6}$ Overall, employee satisfaction, productivity, and health risk scores improve. ${ }^{7,8}$

The association of HRAs and HMPs with health expenditures is unclear because prior studies have found conflicting results. Two studies reported health expenditure reductions after HRA and HMP implementation, 6, 9 while others showed consistent increases over several years of follow-up. ${ }^{4,5,7,10,11}$ A recent multi-site trial found no association between HMPs and health care utilization or expenditures. ${ }^{12}$

The Department of Veterans Affairs (VA) recently completed a trial in which 417 veterans receiving primary care services at one of three VA locations were randomized to complete an HRA followed by telephone-based health coaching, or to complete an HRA without health coaching. ${ }^{13}$ Over 6 months, compared to patients in the HRA-alone group, patients who completed the HRA + coaching program had 2.5 times greater odds of enrolling 
in prevention programs, the trial's main outcome. There was no difference in Framingham risk score at 6 months, a secondary outcome of the trial and its main health outcome.

Short-term health care costs following completion of HRAs and coaching programs in the VA have not been assessed. In this paper, we compare the 6-month total VA health care expenditures of veterans randomized to HRA + coaching to the 6-month expenditures of veterans randomized to HRAalone. We also compare mean expenditures in subgroups of veterans identified using a data-driven method for assessing heterogeneity of treatment effects. The VA is currently preparing to roll out HRA + coaching programs to several more centers, so it is important for the VA to understand the shortterm costs associated with patients who participated in the HRA + coaching and HRA-alone arms. These results can inform the budget impacts of individual participating VA Medical Centers. Our analysis will help the VA predict not only overall future short-term costs, but also the patient subgroups with the highest health care costs following completion of these programs.

\section{METHODS}

\section{Trial Design, Participants, Outcomes, and Covariates}

The ACTIVATE trial was a randomized controlled trial that evaluated enrollment in structured prevention programs among veterans receiving a comprehensive HRA and telephone coaching intervention, compared to veterans receiving the HRA without the coaching intervention (usual care). ${ }^{13}$ The study was conducted from 2014 to 2016 at three VA primary care clinics in Ann Arbor, Michigan, Durham, North Carolina, and Greenville, North Carolina. Veterans were eligible for inclusion if they were enrolled in primary care at one of these three locations and had at least one of the following modifiable risk factors: body mass index (BMI) $\geq 30$, current smoker, or $<$ $150 \mathrm{~min}$ of moderate/vigorous physical activity per week. The protocol was approved by the VA's Central IRB. All eligible patients provided written informed consent prior to enrollment.

All enrolled veterans $(n=417)$ completed the VA's web-based HRA, HealtheLiving Assessment. This HRA, which is available via the VA's patient web portal, ${ }^{14}$ uses a proprietary risk modeling algorithm to determine patients' "health age" based on lifestyle choices, family risk, and biological values, as well as information about the degree to which lifestyle changes can lower their health age. Veterans in the usual care group received a printed copy of their HRA output and were encouraged to discuss their results with their primary care teams. The intervention group received two telephone calls from a trained health coach. The first telephone call occurred 1 week after the baseline interview and consisted of setting goals for enrollment in a prevention program, using a semi-scripted approach.
Using motivational interviewing techniques, coaches reviewed the HRA results, determined patient preferences regarding their modifiable risk factors, discussed strategies for addressing these risk factors, and advised patients on the prevention programs that would be most helpful for achieving their goals. Coaches were instructed to assess patient readiness to enroll in prevention programs and to help patients develop SMART (small, measureable, attainable, relevant, timely) goals. The second telephone call, with the same coach, occurred 1 month later. During this call, coaches determined whether patients had enrolled in one or more prevention programs. If they had not yet enrolled, coaches used motivational interviewing to problem-solve and set new SMART goals. Further details about these telephone calls have been previously published. ${ }^{13}$ After 6 months, the intervention group had higher rates of self-reported enrollment in prevention programs $(51 \%$ vs $29 \%$, odds ratio $(\mathrm{OR})=2.5 ; 95 \%$ confidence interval (CI) 1.7, 3.9; $p<0.0001)$ and greater mean increase in Patient Activation Measure (PAM). ${ }^{13}$ The PAM measures patients' knowledge, skills, beliefs, and confidence in managing their health and has been previously validated. ${ }^{15}$, 16

Sociodemographic characteristics collected at baseline included age, race, ethnicity, gender, marital status, education, employment, and adequacy of income. Clinical characteristics collected at baseline included physical activity, Medical Outcomes Study Sleep Score, ${ }^{17}$ smoking status, alcohol intake, health age, perceived general health, depressive symptoms, pain level, body mass index, diabetes status, use of antihypertensives, blood pressure, total cholesterol, and high-density lipoprotein. Baseline Framingham 10-year cardiovascular risk score $^{18}$ and PAM were also included. Veterans were also asked at baseline about their numeracy skills, health literacy, and computer literacy.

Total VA expenditures were calculated from the day of enrollment in the study to 6 months after enrollment. Expenditure data were obtained from the VINCI Data Manager $^{19}$ and included VA outpatient care, VA outpatient prescription medications, VA inpatient care, and community-based care purchased by the Fee Basis VA program. VA expenditures are derived using a top-down accounting method that disaggregates direct and indirect expenditure estimates from the annual Congressional allocation for each VA product or service. The total expenditure outcome was created by summing expenditures from each component for each veteran. All costs were adjusted to 2016 dollars using the consumer price index from the Bureau of Labor Statistics. ${ }^{20}$ We were unable to obtain cost data on six patients due to patient withdrawal post-randomization, so the analysis included 411 of the 417 enrolled veterans. This analysis was conducted from a VA payer perspective for which total expenditures were the most relevant economic outcome, rather than cost-effectiveness or cost of implementing the intervention. 


\section{Statistical Analysis}

We examined differences in 6-month total VA expenditures between treatment arms, adjusting for study site, using a generalized linear model with a gamma distribution and log link using the SAS procedure PROC GENMOD. The model specification was chosen based upon tests following Manning and Mullahy. ${ }^{21}$

To examine whether veteran subgroups responded favorably to the HRA + coaching intervention or HRA-alone, we performed an exploratory analysis in which we identified subgroups using a data-driven method of model-based recursive partitioning (MoB). MoB splits patients into subgroups based on the full set of available covariates and identifies subgroups based on covariates that provide the greatest discrimination in VA expenditure differences between the two interventions. ${ }^{22}$ A regression model of the treatment effect upon the outcome fit within each subgroup is repeated within each of the resulting subgroups until the best model fit is achieved. MoB yields a regression-based tree, with each terminal node representing a subgroup experiencing different effects of health coaching on total VA expenditures. MoB was implemented using a gamma distribution with a log link via the glmtree function in the $\mathrm{R}$ package partykit version 1.2$4 .^{23} \mathrm{~A}$ total of 28 baseline covariates were included as potential partitioning variables. Results are shown graphically as a regression-based tree and summarized by mean observed costs of patients in each arm for the resulting subgroups. Statistical analyses were performed using SAS for Windows (version 9.4: SAS Institute, Cary, NC) and R version 3.5.2.

\section{RESULTS}

\section{Baseline Patient Characteristics}

The vast majority (85.4\%) of participants were male (Table 1), with a mean age of 55.8 (standard deviation $[\mathrm{SD}]=12.2$ ), mean HRA-generated health age of $60.4(\mathrm{SD}=12.7)$, mean body mass index (BMI) of 33.8 (SD = 6.4), and mean 10-year cardiovascular risk score from the Framingham Risk Score of $22.2 \%$ ( $\mathrm{SD}=16.7$ ). Over a third of veterans were employed part- or full-time $(37.3 \%)$ and $26.5 \%$ of veterans reported inadequate income, poor or fair health $(30.5 \%)$ and tobacco use $(39.7 \%)$. Baseline patient characteristics differ slightly from prior reports from the ACTIVATE trial, ${ }^{13}$ due to a small difference in sample size (417 vs. 411 ).

\section{Cost Outcomes}

Mean unadjusted 6-month expenditures were $\$ 8713$ (SD $=$ $\$ 18,131)$ for veterans randomized to the HRA + coaching arm and were $\$ 9846$ ( $\mathrm{SD}=\$ 16,621$ ) for veterans to the HRAalone arm (Fig. 1). After adjusting for region, mean estimated 6-month total VA expenditures were $\$ 8665$ in the HRA + coaching arm and $\$ 9900$ in the HRA-alone arm, for an absolute difference of $\$ 1235(p=0.25)$.

\section{Subgroup Analysis}

While overall 6-month costs did not differ between the HRA + coaching and HRA-alone arms, several subgroups did exhibit heterogeneity in costs by treatment arm. MoB revealed four subgroups characterized by employment status, sleep habits, and self-reported health (Fig. 1). Patients who were unemployed and had good sleep were stratified into those with good/very good/excellent self-reported health and those with poor/fair self-reported health. Among those with poor/fair health, mean observed total expenditures were higher in the HRA + coaching arm compared to the HRA-alone arm of the trial ( $\$ 12,814$ vs $\$ 7971)$. In contrast, among those with good/ very good/excellent health, mean observed total expenditures were lower in the HRA + coaching arm compared to the HRAalone arm (\$5082 vs $\$ 11,612)$.

Mean observed total expenditures at 6 months were highest among unemployed patients with poor sleep, but similar between the two arms $(\$ 17,602$ in the HRA + coaching arm and $\$ 17,318$ in the HRA-alone arm). Compared to all other subgroups, mean observed total expenditures at 6 months were lowest among employed patients, at \$6146 in the HRA + coaching arm and $\$ 5140$ in the HRA-alone arm.

\section{DISCUSSION}

The average treatment effect of the HRA + coaching intervention on 6-month total VA health care expenditures was null. Over the longer-term, it is possible that cost differences could arise, as patients in the HRA + coaching arm spend more time in prevention programs and take further steps to alter their health risks.

Cost studies of HRAs and HMPs implemented among employees of large corporations have had mixed results, with some finding reductions in health-related spending and some finding no change or even increases in spending. ${ }^{4-11}$ Costs are typically lower when employee participation is high $^{9}$ and when patients' health scores improve from "high-risk" to "low-risk." $10,{ }^{24}$ Most of these studies agree that in order for HRAs and HMPs to have a meaningful impact on health, healthcare utilization, and costs, they must remain in place for several years. While we did not assess differences in health-related costs or utilization over the long term, we did compare costs between two programs of differing intensity, and found no difference.

Results of prior employer-based studies may not be generalizable to the veteran population. The veterans included in our intervention had high rates of unemployment $(62.7 \%)$, inadequate income (26.5\%), poor or fair health $(30.5 \%)$, and tobacco use $(39.7 \%)$. The large majority were male $(85.4 \%)$. Given the poorer health and socioeconomic status, on average, of our patient population, it may be easier for HRAs and health coaching to have a meaningful impact. Improvements in health and reductions in expenditures associated with HRA and coaching programs may also be seen sooner than in prior studies. Our results, however, suggest that 6 months' worth of data are not 
Table 1 Baseline Patient Characteristics

\begin{tabular}{|c|c|c|c|}
\hline & $\begin{array}{l}\text { Overall } \\
N=411\end{array}$ & $\begin{array}{l}\text { Intervention: } \\
\text { HRA + coaching } \\
N=203\end{array}$ & $\begin{array}{l}\text { Control: } \\
\text { HRA-alone } \\
N=\mathbf{2 0 8}\end{array}$ \\
\hline \multicolumn{4}{|l|}{ Demographics } \\
\hline Age, mean (SD) & $55.8(12.2)$ & $55.2(12.8)$ & $56.4(11.7)$ \\
\hline Non-Hispanic white race, $n(\%)$ & $198(48.2)$ & $94(46.3)$ & $104(50.0)$ \\
\hline Male gender, $n(\%)$ & $351(85.4)$ & $167(82.3)$ & $184(88.5)$ \\
\hline Married/living as married, $n(\%)$ & $210(51.1)$ & $93(45.8)$ & $117(56.3)$ \\
\hline \multicolumn{4}{|l|}{ Education, $n(\%)$} \\
\hline High school or less & $73(17.8)$ & $37(18.2)$ & $36(17.3)$ \\
\hline Some college, associate's degree, or trade school & $236(57.4)$ & $109(53.7)$ & $127(61.1)$ \\
\hline Bachelor's degree or higher & $102(24.8)$ & $57(28.1)$ & $45(21.6)$ \\
\hline Employed full/part time, $n(\%)$ & $153(37.3)$ & $79(39.1)$ & $74(35.6)$ \\
\hline Inadequate income, $n(\%)$ & $109(26.5)$ & $56(27.6)$ & $53(25.5)$ \\
\hline \multicolumn{4}{|l|}{ Literacy } \\
\hline \multicolumn{4}{|l|}{ Assistance required for reading, $n(\%)$} \\
\hline Never & $288(70.1)$ & $145(71.4)$ & $143(68.8)$ \\
\hline Rarely & $76(18.5)$ & $39(19.2)$ & $37(17.8)$ \\
\hline Sometimes/often/always & 47 (11.4) & $19(9.4)$ & $28(13.5)$ \\
\hline Average of 3 numeracy variables*, mean (SD) & $4.6(1.2)$ & $4.6(1.2)$ & $4.6(1.2)$ \\
\hline \multicolumn{4}{|l|}{ Computer literacy, $n(\%)$} \\
\hline Do not use computer & $23(5.6)$ & $14(6.9)$ & $9(4.3)$ \\
\hline Basic & $79(19.2)$ & $41(20.2)$ & $38(18.3)$ \\
\hline Moderate & $151(36.7)$ & $72(35.5)$ & $79(38.0)$ \\
\hline Advanced & $109(26.5)$ & $53(26.1)$ & $56(26.9)$ \\
\hline Expert & 49 (11.9) & $23(11.3)$ & $26(12.5)$ \\
\hline \multicolumn{4}{|l|}{ Health habits } \\
\hline Minutes of physical activity in past week, median (IQR) & $140.0(380.0)$ & $150.0(380.0)$ & $125.0(380.0)$ \\
\hline MOS-6 Sleep Scale Score, mean (SD) & $61.1(21.7)$ & $61.4(21.3)$ & $60.9(22.1)$ \\
\hline Current tobacco use, $n(\%)$ & $163(39.7)$ & $88(43.3)$ & $75(36.1)$ \\
\hline \multicolumn{4}{|l|}{ Alcohol consumption, $n(\%)$} \\
\hline Never & $166(40.4)$ & $71(35.0)$ & $95(45.7)$ \\
\hline Monthly or less & $93(22.6)$ & $50(24.6)$ & $43(20.7)$ \\
\hline 2-4 times a month & 57 (13.9) & $25(12.3)$ & $32(15.4)$ \\
\hline $2-3$ times a week & $58(14.1)$ & $31(15.3)$ & $27(13.0)$ \\
\hline 4 or more times a week & $37(9.0)$ & $26(12.8)$ & $11(5.3)$ \\
\hline \multicolumn{4}{|l|}{ Clinical characteristics } \\
\hline HLA health age ${ }^{\dagger}$, mean (SD) & $60.4(12.7)$ & $60.0(13.0)$ & $60.7(12.4)$ \\
\hline Difference between Health age and actual age, mean (SD) & $4.6(5.7)$ & $4.8(5.6)$ & $4.4(5.9)$ \\
\hline \multicolumn{4}{|l|}{ Perceived general health, $n(\%)$} \\
\hline Excellent & $23(5.6)$ & $13(6.4)$ & $10(4.8)$ \\
\hline Very good & $98(23.9)$ & $43(21.2)$ & $55(26.6)$ \\
\hline Good & $164(40.0)$ & $83(40.9)$ & $81(39.1)$ \\
\hline Fair & $96(23.4)$ & $47(23.2)$ & 49 (23.7) \\
\hline Poor & $29(7.1)$ & $17(8.4)$ & $12(5.8)$ \\
\hline PHQ-8 Total Score, mean (SD) & $7.1(5.4)$ & $6.8(5.4)$ & $7.4(5.5)$ \\
\hline Pain in past week ${ }^{\dagger}$ mean (SD) & $4.6(2.7)$ & $4.4(2.7)$ & $4.7(2.7)$ \\
\hline Body mass index, mean (SD) & $33.8(6.4)$ & $33.6(6.6)$ & $33.9(6.2)$ \\
\hline Diabetes diagnosis, $n(\%)$ & $113(27.5)$ & $52(25.6)$ & $61(29.3)$ \\
\hline Taking blood pressure medications, $n(\%)$ & $254(61.8)$ & $126(62.1)$ & $128(61.5)$ \\
\hline Average systolic blood pressure $(\mathrm{mmHg})^{\S}$, mean $(\mathrm{SD})$ & $129.8(15.4)$ & $129.6(15.7)$ & $130.1(15.1)$ \\
\hline Total cholesterol (mg/dL), mean (SD) & $179.8(42.0)$ & $179.6(42.1)$ & $180.0(42.0)$ \\
\hline High-density lipoprotein $(\mathrm{mg} / \mathrm{dL})$, mean $(\mathrm{SD})$ & $45.8(14.0)$ & $46.8(14.7)$ & $44.8(13.3)$ \\
\hline PAM score, mean (SD) & $61.6(12.6)$ & $62.6(12.8)$ & $60.6(12.3)$ \\
\hline \multirow{2}{*}{\multicolumn{4}{|c|}{ Total number of study inclusion criteria met", $n(\%)$}} \\
\hline & & & \\
\hline 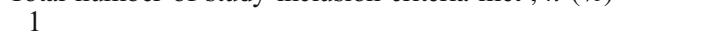 & $170(41.4)$ & $84(41.4)$ & $86(41.3)$ \\
\hline 2 & $197(47.9)$ & $99(48.8)$ & $98(47.1)$ \\
\hline 3 & $44(10.7)$ & $20(9.9)$ & $24(11.5)$ \\
\hline
\end{tabular}

SD standard deviation, IQR interquartile range, HRA health risk assessment, HLA HealtheLiving Assessment, MOS Medical Outcomes Study, PHQ Patient Health Questionnaire, PAM Patient Activation Measure

Missing data: Employment (1), health age (1), difference between health age and age (1), general health (1), cholesterol (6), high-density lipoprotein (6), Framingham (8)

*Numeracy variables: skill with fractions, skill with percentages, and usefulness of numerical information in making health decisions; each variable is on a 1-6 scale, with a value of 1 anchoring "not at all good" and 6 anchoring "extremely good"

${ }^{\prime} H L A$ health age: The HLA uses a proprietary risk modeling algorithm to determine patients' "health age" based on lifestyle choices, family risk, and biological values, as well as information about the degree to which lifestyle changes can lower their "health age"

${ }^{7}$ Pain is measured on a $0-10$ scale, with 0 representing no pain

${ }^{s}$ Average of two systolic blood pressure measurements

"To be included in the study, veterans had to have at least one of the following modifiable risk factors: body mass index (BMI) $\geq 30$, current smoker, or

$<150$ min of moderate/vigorous physical activity per week 


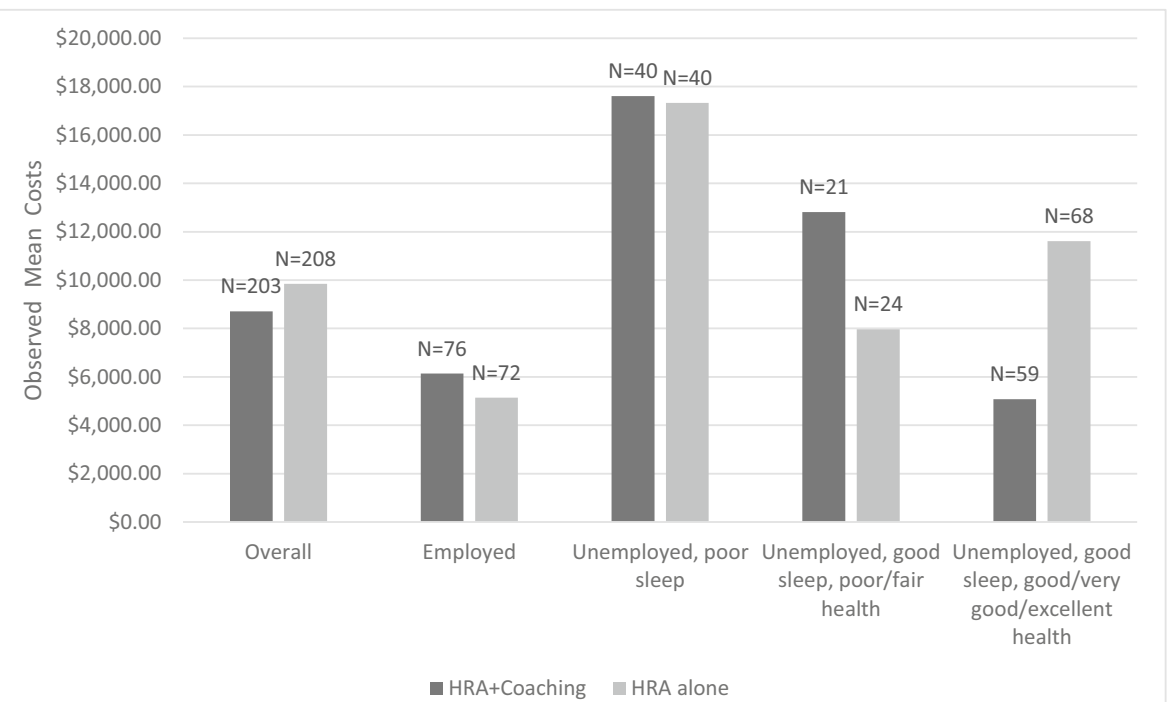

Figure 1 Overall observed costs of health risk assessment (HRA) + coaching and HRA-alone at 6 months were similar, but treatment effects were heterogeneous. Subgroups of trial participants with differential treatment effects were derived using model-based recursive partitioning (MoB). We used the default value of statistical significance for the fluctuation tests (alpha = 0.05); post pruned via AIC fit index; set minimum node sample size as 40; and specified max LM-type test as the fluctuation test for ordered factor variables. All other control parameters were kept at their default values. The effective sample size used in the model-based recursive partitioning analysis was reduced to 400 due to missing data in baseline covariates. MoB generated a tree based upon three variables. Employment status was the strongest predictor of treatment response. An additional split on the MOS-6 Sleep Scale Score occurred within those not employed full or part time, and those with "good sleep" had an additional split based upon self-rated health.

enough. As it works to implement our HRA + coaching program at several more primary care clinics, the VA will need to ensure that programs remain in place for at least 1 year, and ideally more. The VA should also invest in collecting clinical, utilization, and cost data on participating patients to improve our understanding of the programs' efficacy and long-term financial viability.

Several previous evaluations of HRA programs have reported finding that a few individuals in their cohorts had extremely high costs. ${ }^{4,9}$ These outliers were typically removed from cost analyses to avoid skewing cost outcomes for one of their comparison groups. If the VA is to disseminate HRA + coaching programs widely, however, it should not remove these outliers from its cost evaluations. To the contrary, these programs should specifically target patients with high rates of health care utilization, with the goal of improving their health and, over the long term, reducing their utilization and costs for the benefit of the health system as a whole.

Our finding that the average treatment effect was null masked meaningful differences in patient subgroups examined in exploratory analysis. The higher expenditures associated with unemployed patients compared to employed patients were expected, as people who are unemployed tend to have a higher prevalence of disabilities and other costly conditions. ${ }^{25}$ In the unemployed group, veterans who reported poor sleep had higher costs, regardless of treatment arm. This finding makes sense - chronically poor and insufficient sleep has been linked to higher rates of cardiovascular disease, immunosuppression, and obesity, all of which are associated with high costs. ${ }^{26-28}$ HRA + coaching and HRA-alone had differential effects among patients who were unemployed, with good sleep, and poor or fair perceived health, with patients randomized to HRA + coaching accruing higher short-term costs. It is possible that coaching increased these patients' awareness of both their current and future health risks, thus motivating them to increase their health care resource utilization and, potentially, enroll in appropriate prevention programs. In the long run, if the prevention programs are successful, we may expect to see a reduction in expenditures in this group, as these patients become healthier. This exploratory subgroup analysis consisted of relatively small numbers of observations, but it did reveal some interesting results that should be further evaluated in larger samples. It will be particularly important to observe VA expenditures over one or more years to evaluate whether these cost differences persist.

Several limitations must be acknowledged. First, capture of community-based care purchased by VA may be slightly under-counted because there is a lag between the time of care and claims processing. Second, results may not generalize beyond veterans. Third, the subgroup results are hypothesisgenerating and not definitive results.

In conclusion, working with health coaches to set actionable behavior change goals following HRA completion did not reduce short-term health expenditures compared to completing and receiving HRA results alone. As the VA disseminates this program to other VHA primary care clinics, health care expenditures and 
clinical outcomes beyond 6 months should be examined so that the VA can assess whether HRA + coaching interventions are sustainable in the long run.

Acknowledgments: This work was supported by the HSR\&D funding (CRE 12-306, RCS 10-391) and by the Durham Center of Innovation to Accelerate Discovery and Practice Transformation (ADAPT), (CIN 13-410) at the Durham VA Health Care System. The views represented in this article represent those of the authors and not those of the VA or the United States Government.

Corresponding Author: Matthew L. Maciejewski, PhD; Durham Center of Innovation to Accelerate Discovery and Practice Transformation (ADAPT), Durham Veterans Affairs Health Care System. Durham, NC, USA (e-mail: matthew.maciejewski@va.gov).

\section{Compliance with ethical standards:}

Conflict of interest: Dr. Maciejewski owns Amgen stock due to his spouse's employment. All other authors have no conflicts.

\section{REFERENCES}

1. Fielding JE. Frequency of health risk assessment activities at U.S worksites. Am J Prev Med. 1989; 5: 73-81.

2. Aldana SG. Financial impact of health promotion programs: a comprehensive review of the literature. Am J Health Promot AJHP. 2001; 15: 296-320.

3. Haas JS, Baer HJ, Eibensteiner K, et al. A Cluster Randomized Trial of a Personalized Multi-Condition Risk Assessment in Primary Care. Am J Prev Med. 2017; 52: 100-5.

4. Sieck CJ, Dembe AE. A 3-Year Assessment of the Effects of a SelfAdministered Health Risk Assessment on Health Care Utilization, Costs, and Health Risks: J Occup Environ Med. 2014; 56: 1284-90.

5. Parkinson MD, Peele PB, Keyser DJ, Liu Y, Doyle S. UPMC MyHealth: managing the health and costs of U.S. healthcare workers. Am J Prev Med. 2014; 47: 403-10.

6. Ozminkowski RJ, Ling $\mathbf{D}$, Goetzel RZ, et al. Long-Term Impact of Johnson \& Johnson's Health \& Wellness Program on Health Care Utilization and Expenditures: J Occup Environ Med. 2002; 44: 21-9.

7. Musich S, McCalister T, Wang S, Hawkins K. An Evaluation of the Well at Dell Health Management Program: Health Risk Change and Financial Return on Investment. Am J Health Promot. 2015; 29: 147-57.

8. Fries JF, Bloch DA, Harrington H, Richardson N, Beck R. Two-year results of a randomized controlled trial of a health promotion program in a retiree population: the Bank of America Study. Am J Med. 1993; 94: 455-62.

9. Serxner SA, Gold DB, Grossmeier JJ, Anderson DR. The Relationship Between Health Promotion Program Participation and Medical Costs:: A Dose Response. J Occup Environ Med. 2003; 45: 1196-200.

10. Burton WN, Chen C-Y, Li X, Schultz AB, Kasiarz D, Edington DW. Evaluation of a Comprehensive Employee Wellness Program at an Organization With a Consumer-Directed Health Plan: J Occup Environ Med. 2014; 56: 347-53.
11. Mattke S, Serxner SA, Zakowski SL, Jain AK, Gold DB. Impact of 2 employer-sponsored population health management programs on medical care cost and utilization. Am J Manag Care. 2009; 15: 113-20.

12. Song Z, Baicker K. Effect of a Workplace Wellness Program on Employee Health and Economic Outcomes: A Randomized Clinical Trial. JAMA 2019; 321: 1491-501.

13. Oddone EZ, Gierisch JM, Sanders LL, et al. A Coaching by Telephone Intervention on Engaging Patients to Address Modifiable Cardiovascular Risk Factors: a Randomized Controlled Trial. J Gen Intern Med 2018; 33 1487-94.

14. Veterans Administration. MyHealtheVet. https://www.myhealth.va.gov/ mhv-portal-web/home. Accessed 2/1/2018

15. Hibbard JH, Mahoney ER, Stockard J, Tusler M. Development and testing of a short form of the patient activation measure. Health Serv Res. 2005; 40: 1918-30.

16. Hibbard JH, Mahoney ER, Stock R, Tusler M. Do increases in patient activation result in improved self-management behaviors? Health Serv Res. 2007; 42: 1443-63.

17. Stewart A, Ware J, Brook R, Davies A. Conceptualization and measurement of physical health for adults in the Health Insurance Study, Vol. II, The RAND Corporation (R-1987/2-HEW), Santa Monica, Calif. 1978.

18. Wilson PW, D'Agostino RB, Levy D, Belanger AM, Silbershatz $\mathbf{H}$, Kannel WB. Prediction of coronary heart disease using risk factor categories. Circulation. 1998; 97: 1837-47.

19. U.S. Department of Veterans Affairs. VA Informatics and Computing Infrastructure (VINCI), VA HSR HIR 08-204. 2008 https://vaww.VINCI. med.va.gov (accessed Oct 19, 2018).

20. Consumer Price Index (CPI) Databases. U. S. Dep. Labor Bur. Labor Stat. https://www.bls.gov/cpi/data.htm (accessed Dec 17, 2018).

21. Manning WG, Mullahy J. Estimating log models: to transform or not to transform? J Health Econ. 2001; 20: 461-94.

22. Seibold H, Zeileis A, Hothorn T. Model-Based Recursive Partitioning for Subgroup Analyses. Int J Biostat. 2016; 12: 45-63.

23. Hothorn T, Zeileis A. partykit: A Modular Toolkit for Recursive Partytioning in R. J Mach Learn Res. 2015; 16: 3905-9.

24. Schultz AB, Lu C, Barnett TE, et al. Influence of participation in a worksite health-promotion program on disability days. J Occup Environ Med. 2002; 44: 776-80.

25. Singh GK, Daus GP, Allender M, et al. Social Determinants of Health in the United States: Addressing Major Health Inequality Trends for the Nation, 1935-2016. Int J MCH AIDS 2017; 6: 139-64.

26. Besedovsky L, Lange T, Born J. Sleep and immune function. Pflugers Arch. 2012; 463: 121-37.

27. Hoevenaar-Blom MP, Spijkerman AMW, Kromhout D, Verschuren WMM. Sufficient sleep duration contributes to lower cardiovascular disease risk in addition to four traditional lifestyle factors: the MORGEN study. Eur J Prev Cardiol. 2014; 21: 1367-75.

28. Deng H-B, Tam T, Zee BC-Y, et al. Short Sleep Duration Increases Metabolic Impact in Healthy Adults: A Population-Based Cohort Study. Sleep. 2017; 40. https://doi.org/10.1093/sleep/zsx130.

Publisher's Note Springer Nature remains neutral with regard to jurisdictional claims in published maps and institutional affiliations. 